\title{
Reactions of chiral n-tert-butanesulfinyl imines with ethyl nitroacetate
}

\begin{abstract}
The reaction of chiral N-tert-butanesulfinyl imines with ethyl nitroacetate under substoichiometric amounts of a base or an acid is studied. The best results are obtained using 0.1 equivalents of sodium methoxide in methanol. The addition takes place with high facial diastereoselectivity at the $\mathrm{C}-\mathrm{N}$ stereocenter giving rise to the corresponding $\beta$-amino- $\alpha$-nitroesters as an almost 1:1 diastereomeric mixture due to the easy epimerization at the $\alpha$-position of the nitroester under the reaction conditions.
\end{abstract}

Keywords: chiral imines, diastereoselective additions, aza-henry reactions, nitrocompounds

\author{
Volume I Issue 6 - 2017 \\ Nítida Navarro,' Juan Alberto Sirvent, ${ }^{1,2,3}$ \\ Francisco Foubelo, ${ }^{1,2,3}$ Miguel Yus ${ }^{1,3}$ \\ 'Departamento de Química Orgánica, Universidad de Alicante, \\ Spain \\ ${ }^{2}$ Instituto de Síntesis Orgánica (ISO), Universidad de Alicante, \\ Spain \\ ${ }^{3}$ Centro de Innovación en Química Avanzada (ORFEO-CINQA), \\ Universidad de Alicante, Spain
}

\begin{abstract}
Correspondence: Miguel Yus, Departamento de Química Orgánica, Facultad de Ciencias, Universidad de Alicante, Apdo. 99, 03080 Alicante, Spain, Tel +43965 903548, Email yus@ua.es

Francisco Foubelo, Departamento de Química Orgánica, Facultad de Ciencias, Universidad de Alicante,Apdo. 99, 03080 Alicante, Spain, Tel +4396 5909 672, Email foubelo@ua.es

Received: October 28, 2017 | Published: November 24, 2017
\end{abstract}

\section{Introduction}

The stereoselective synthesis of complex molecules of natural or artificial origin is one of the most important tasks for organic chemists. ${ }^{1}$ Of particular importance is the construction of lineal chains bearing several stereocenters: for this purpose, the nucleophilic addition of nitroalkanes to imines, the so-called aza-Henry or nitro-Mannich reaction, plays a pivotal role because together with the formation of a new carbon-carbon bond two stereogenic centres are generated bearing two different nitrogenated functional groups (Figure 1). ${ }^{2}$

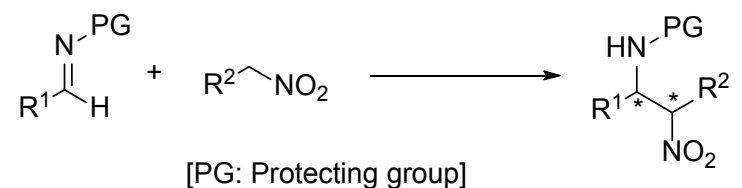

Figure I The aza-Henry reaction.

One special group for protecting imines is the sulfinyl one (PG: $\mathrm{RSO}$ ) because in this case this moiety not only activates the $\mathrm{C}=\mathrm{N}$ group but also provides a chiral information allowing a possible asymmetric induction. ${ }^{3,4}$ In our research group, and inspired by the work of García-Ruano with p-tolylsulfinyl imines, ${ }^{5}$ we have studied the reaction of $\mathrm{N}$-tert-butanesulfinyl imines with nitromethane or nitroethane to yield protected $\beta$-nitro amines, easily transformed into $\alpha$-amino acids or $\alpha$-amino ketones by standard methodologies. ${ }^{6}$ Finally, using 4-nitrobutanoates, the addition products were directly transformed into chiral nitropiperidines, interesting precursors of a variety of heterocycles with biological activity. ${ }^{7}$ In the present paper we report the reaction between N-tert-butanesulfinyl imines with ethyl nitroacetate to give molecules bearing three contiguous functionalities and two new stereocentres.

\section{Results and discussion}

The starting (E)-N-tert-butanesulfinyl aldimines 1 were easily prepared by reaction of the corresponding aldehydes with commercially available (R)-tert-butanesulfinamide in the presence of titanium tetraisopropoxide at room temperature following the already reported methodology.

In order to optimize the reaction conditions, and as a model reaction, the imine $1 \mathrm{~d}$ derived from 3-phenylpropanal was allowed to react with ethyl nitroacetate 2 under either basic or acidic conditions yielding a diastereomeric mixture of product $3 \mathrm{~d}$ (Table 1). As it can be deduced from Table 1, Entry 6 shows the best result using sodium ethoxide and an excess of ethyl nitroacetate. In addition, under acidic conditions (Table 1, Entry 7), using a substoichiometric amount of triflic acid, the reaction proceeded with modest yield. In the absence of a basic or acidic medium a lower yield was obtained (Table 1, Entry 8).

With the best reaction conditions in hand, different aldimines 1 were assayed using both basic (Method A) or acidic (Method B) medium (Table 2). Thus, in general, basic conditions gave better results than acidic ones, steric effects playing an important role in the reaction: the best yields were obtained for imines without substitution at the $\alpha$-position (compare Entries 3 and 5 in Table 2). On the other hand, the reaction with either aromatic imines $\left(1\right.$ with $\left.\mathrm{R}^{1}=\mathrm{Ph}\right)$ or ethyl or methyl 3-nitropropane under the assayed reaction conditions shown in Table 1, failed.

The stereochemistry of diastereomers 3 (Table 2) could not be assigned by NMR data, but the corresponding ratio was determined by this technique. In any case, a suitable monocrystal was not obtained in order to perform an X-ray structure determination. Anyhow, the obtained stereochemistry could be explained accepting that the addition took place through the cyclic transition state 4 , in which the cation (or the hydrogen atom) is coordinated to the nitro group and the nitrogen and sulphur atoms of the imine (Figure 2).

Despite giving a diastereomeric mixture of products 3, these can 
be easily transformed into enantiomerically pure $\alpha$-ketoesters by a elaborated. ${ }^{7}$ simple base-acid tandem treatment, which can be further synthetically

Table I Optimization of the reaction conditions

\begin{tabular}{lll}
\hline Entry & Reaction conditions & 3d (\%)b \\
\hline I & $\mathrm{NaHCO}_{3}\left(0.4 \mathrm{mmol}, 2\right.$ equiv), $60^{\circ} \mathrm{C}, 72 \mathrm{~h}$ & -- \\
2 & $\mathrm{NaOH}\left(0.075 \mathrm{mmol}, 0.38\right.$ equiv), $40^{\circ} \mathrm{C}, 24 \mathrm{~h}$ & -- \\
3 & $\mathrm{Al}_{2} \mathrm{O}_{3}$ básica $(750 \mathrm{mg}, 7.35 \mathrm{mmol}), 23^{\circ} \mathrm{C}, 24 \mathrm{~h}$ & $--\mathrm{C}$ \\
4 & $2 \mathrm{M} \mathrm{NaOH}\left(0.02 \mathrm{~mL}, 0.04 \mathrm{mmol}, 0.2\right.$ equiv), $23^{\circ} \mathrm{C}, 16 \mathrm{~h}$ & $<10 \mathrm{~d}$ \\
5 & $2 \mathrm{M} \mathrm{NaOH}\left(0.02 \mathrm{~mL}, 0.04 \mathrm{mmol}, 0.2\right.$ equiv), $60^{\circ} \mathrm{C}, 16 \mathrm{~h}$ & 44 \\
6 & $\mathrm{IM} \mathrm{NaOMe}\left(0.02 \mathrm{~mL}, 0.02 \mathrm{mmol}, 0.1\right.$ equiv), $60^{\circ} \mathrm{C}, 16 \mathrm{~h}$ & 70 \\
7 & $\mathrm{TfOH}\left(0.02 \mathrm{mmol}, 0.1\right.$ equiv), $60^{\circ} \mathrm{C}, 16 \mathrm{~h}$ & 46 \\
8 & $60^{\circ} \mathrm{C}, 70 \mathrm{~h}$ & $3 \mathrm{le}$ \\
\hline
\end{tabular}

a. All reactions were carried out using $0.2 \mathrm{mmol}$ of the starting aldimines Id and $0.8 \mathrm{mmol}$ of ethyl nitroacetate (2).

b. Isolated yield after purification by column chromatography (silica gel, hexane/ethyl acetate) based on the starting aldimine Id.

c. A $15 \%$ of the corresponding decarboxylated product was the only isolated one after chromatography.

d. Determined by 'H-NMR from the reaction crude.

e. A 8:I ratio of $3 \mathrm{~d}: I \mathrm{~d}$ was determined by 'H-NMR from the reaction crude.<smiles>CCOC(=O)C[N+](=O)[O-]</smiles>

Table 2 Synthesis of $\beta$-amino- $\alpha$-nitroesters 3

\begin{tabular}{|c|c|c|c|c|c|c|c|}
\hline \multicolumn{4}{|c|}{ Starting imine I } & \multicolumn{4}{|c|}{ Reaction product 3} \\
\hline Entry & No. & $\mathbf{R}^{\prime}$ & $\mathbf{R}^{2}$ & Method & No. & Structure & Yield $^{a}(d r)^{b}$ \\
\hline I & la & $\mathrm{CH}_{3}\left(\mathrm{CH}_{2}\right)_{7}$ & $\mathrm{H}$ & A & $3 a$ & & $61 \%(57: 43)$ \\
\hline 2 & la & $\mathrm{CH}_{3}\left(\mathrm{CH}_{2}\right)_{7}$ & $\mathrm{H}$ & B & $3 a$ & & $48 \%(58: 42)$ \\
\hline 3 & $\mathrm{Ib}$ & $i-P r$ & $\mathrm{H}$ & A & $3 b$ & & $40 \%(62: 38)$ \\
\hline 4 & $\mathrm{Ib}$ & i-Pr & $\mathrm{H}$ & B & $3 b$ & & $28 \%(61: 39)$ \\
\hline 5 & Ic & i-Bu & $\mathrm{H}$ & A & $3 c$ & & $65 \%(53: 47)$ \\
\hline
\end{tabular}




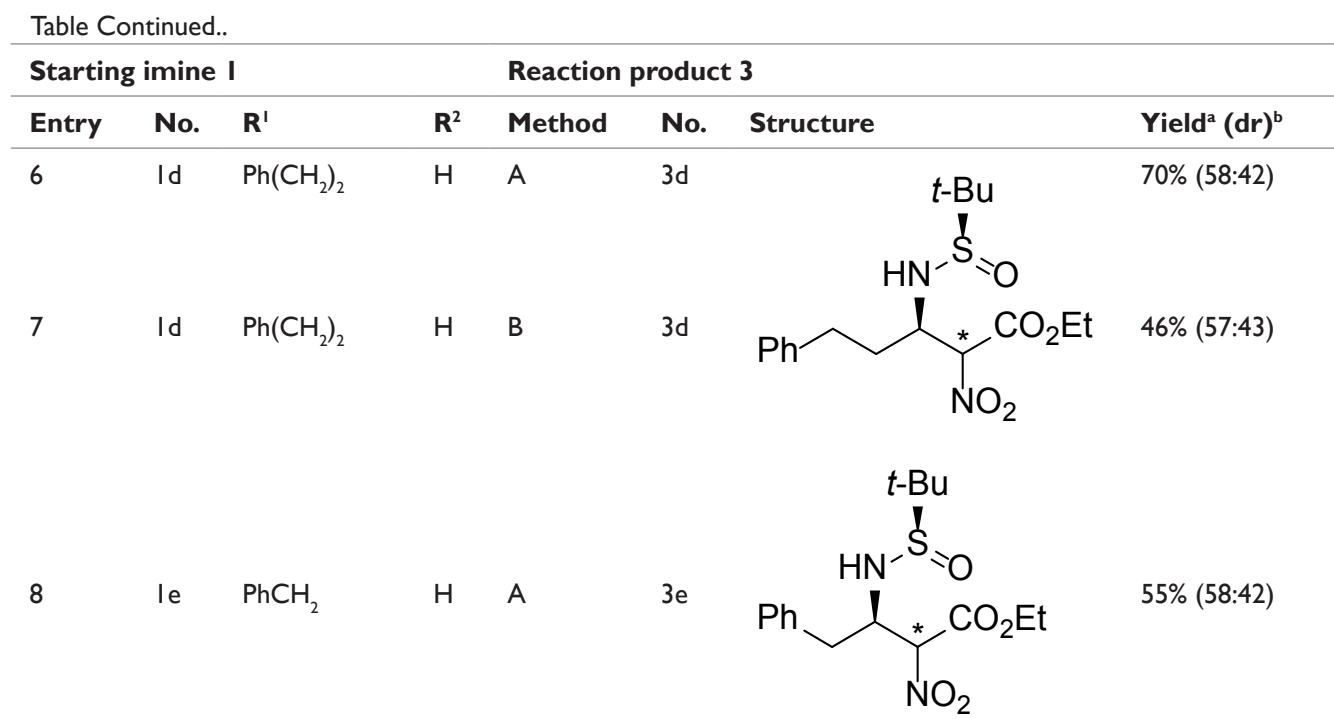

a. Isolated yield after purification by column chromatography (silica gel, hexane/EtOAc) based on the starting imine 3 .

b. Determined by 'H-NMR from the reaction crude.

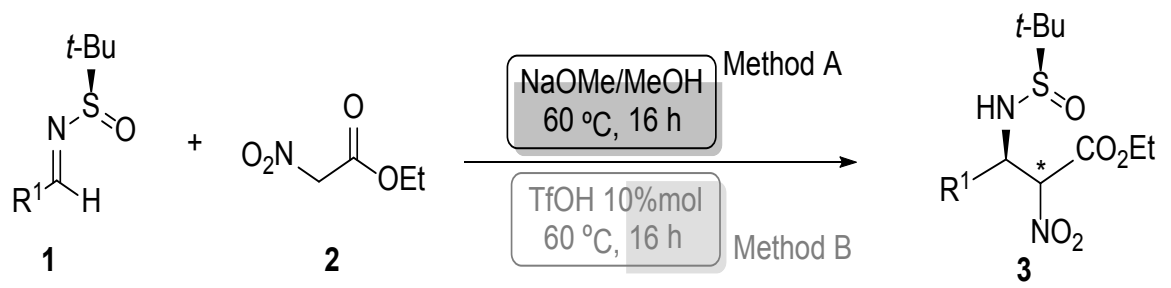

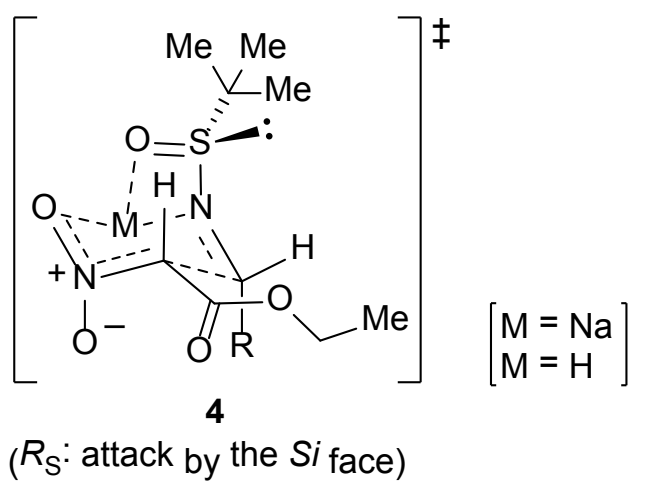

Figure 2 Proposed transition state for the reaction of I with 2.

\section{Experimental}

For general information see reference. ${ }^{7}$

\section{General procedure for the preparation of compound 3}

Method A: To a mixture of the corresponding imine $1(0.2 \mathrm{mmol})$ and ethyl nitroacetate $2(0.106 \mathrm{~g}, 0.8 \mathrm{mmol})$ was added a $1 \mathrm{M}$ methanol solution of sodium methoxide $(0.02 \mathrm{~mL}, 0.2 \mathrm{mmol})$ and it was stirred for $16 \mathrm{~h}$ at $60^{\circ} \mathrm{C}$. The resulting mixture was hydrolysed with water $(15 \mathrm{~mL})$ and extracted with EtOAc $(15 \mathrm{~mL})$. The organic layer was neutralized with $2 \mathrm{M}$ hydrochloric acid and extracted with EtOAc $(3 \times 15 \mathrm{~mL})$, the organic phase being dried with $\mathrm{MgSO}_{4}$ and evaporated under reduced pressure (15 Torr). The resulting residue was purified by flash chromatography (silica gel, hexane: EtOAc) to give products 3 .

Method B: To a mixture of the imine $1(0.2 \mathrm{mmol})$ and ethyl nitroa- cetate $(0.106 \mathrm{~g}, 0.8 \mathrm{mmol})$ was added triflic acid $(3 \mathrm{mg}, 0.02 \mathrm{mmol})$ and the mixture was stirred for $16 \mathrm{~h}$ at $60^{\circ} \mathrm{C}$. After hydrolysing with water $(15 \mathrm{~mL})$ the reaction mixture was worked-up as it was described in Method A. Yields and diastereomeric ratios are given in Table 2. Physical and spectroscopic data follow.

\section{Ethyl (2R*,3R,RS)-3-amino-N-(tert-butanesulfinyl)- -2-nitroundecanoate (3a)}

Yellow oil; $\mathrm{R}_{\mathrm{F}} 0.74$ (hexane/AcOEt 2:1); IR (film) v 2957, 2827, $2856,1749,1562,1265,1019,735 \mathrm{~cm}^{-1} ;{ }^{1} \mathrm{H}-\mathrm{NMR}\left(300 \mathrm{MHz}, \mathrm{CDCl}_{3}\right) \delta$ $0.89\left(6 \mathrm{H}, \mathrm{t}, \mathrm{J}=6.9,2 \times \mathrm{CH}_{3}\right), 1.17\left[9 \mathrm{H}, \mathrm{s},\left(\mathrm{CH}_{3}\right)_{3}\right], 1.18\left[9 \mathrm{H}, \mathrm{s},\left(\mathrm{CH}_{3}\right)_{3}\right]$, $1.20-2.00\left(34 \mathrm{H}, \mathrm{m}, 2 \times \mathrm{CH}_{3}, 14 \times \mathrm{CH}_{2}\right), 4.04-4.42\left(8 \mathrm{H}, \mathrm{m}, 2 \times \mathrm{CH}_{2}\right.$, $2 \times \mathrm{CH}, 2 \times \mathrm{NH}), 5.20(1 \mathrm{H}, \mathrm{d}, \mathrm{J}=5.3 \mathrm{~Hz}, \mathrm{CH}), 5.31(1 \mathrm{H}, \mathrm{d}, \mathrm{J}=5.3 \mathrm{~Hz}$, $\mathrm{CH}) ;{ }^{13} \mathrm{C}-\mathrm{NMR}\left(100 \mathrm{MHz}, \mathrm{CDCl}_{3}\right) \delta 13.8,13.85,14.0,14.1,22.4,22.5$ $\left(\mathrm{CH}_{3}\right), 22.6,25.9,28.9,29.0,29.05,29.1,29.2,31.7,32.9,33.7\left(\mathrm{CH}_{2}\right)$, 56.7, $56.8(\mathrm{C}), 58.3,58.4(\mathrm{CH}), 63.0,63.2\left(\mathrm{CH}_{2}\right), 89.1,90.5(\mathrm{CH})$, 162.7, 162.9 (C); LRMS (EI) m/z 208 (M+C8H17-t-Bu, 6), 142 (15), 137 (15), 135 (30), 122 (21), 113 (32), 95 (33), 81 (44), 69 (49), 67 (47), 57 (100), 55 (81) ( ).

\section{Ethyl (2R*,3R,RS)-3-amino-N-(tert-butanesulfinyl)- -4-methyl-2-nitropentanoate (3b)}

Yellow oil; $\mathrm{R}_{\mathrm{F}} 0.48$ (hexane/AcOEt 2:1); IR (film) v 2966, 2946, 2868, 1749, 1562, 1266, 1071, $1013 \mathrm{~cm}^{-1}$; ${ }^{1} \mathrm{H}-\mathrm{NMR}(300 \mathrm{MHz}$, $\left.\mathrm{CDCl}_{3}\right) \delta 1.03\left(6 \mathrm{H}, \mathrm{d}, \mathrm{J}=6.8,2 \times \mathrm{CH}_{3}\right), 1.09\left(6 \mathrm{H}, \mathrm{d}, \mathrm{J}=6.8,2 \times \mathrm{CH}_{3}\right)$, $1.17\left[9 \mathrm{H}, \mathrm{s},\left(\mathrm{CH}_{3}\right)_{3}\right], 1.19\left[9 \mathrm{H}, \mathrm{s},\left(\mathrm{CH}_{3}\right)_{3}\right], 1.33\left(3 \mathrm{H}, \mathrm{t}, \mathrm{J}=7.1 \mathrm{~Hz}, \mathrm{CH}_{3}\right)$, $1.35\left(3 \mathrm{H}, \mathrm{t}, \mathrm{J}=7.1 \mathrm{~Hz}, \mathrm{CH}_{3}\right), 1.88-1.95(2 \mathrm{H}, \mathrm{m}, 2 \times \mathrm{CH}), 3.86-4.35(8 \mathrm{H}$, $\left.\mathrm{m}, 2 \times \mathrm{CH}_{2}, 2 \times \mathrm{CH}, 2 \times \mathrm{NH}\right), 5.16(1 \mathrm{H}, \mathrm{d}, \mathrm{J}=5.0 \mathrm{~Hz}, \mathrm{CH}), 5.33(1 \mathrm{H}, \mathrm{d}$, $\mathrm{J}=5.0 \mathrm{~Hz}, \mathrm{CH}) ;{ }^{13} \mathrm{C}-\mathrm{NMR}\left(100 \mathrm{MHz}, \mathrm{CDCl}_{3}\right) \delta 13.8,18.6,19,6,19.7$, 19.8, 22.5, $22.6\left(\mathrm{CH}_{3}\right), 31.1,31.8(\mathrm{CH}), 56.8,56.9(\mathrm{C}), 63.0,63.2$ 
$\left(\mathrm{CH}_{2}\right), 63.3,64.3(\mathrm{CH}), 87.9,89.9(\mathrm{CH}), 163.0(\mathrm{C})$; LRMS (EI) m/ z207 ( $\left.\mathrm{M}^{+}-\mathrm{C}_{4} \mathrm{H}_{8}-\mathrm{EtO}, 6\right), 172$ (14), 144 (56), 126 (17), 108 (29), 97 (65), 82 (43), 67 (100) (Figure 4 ).

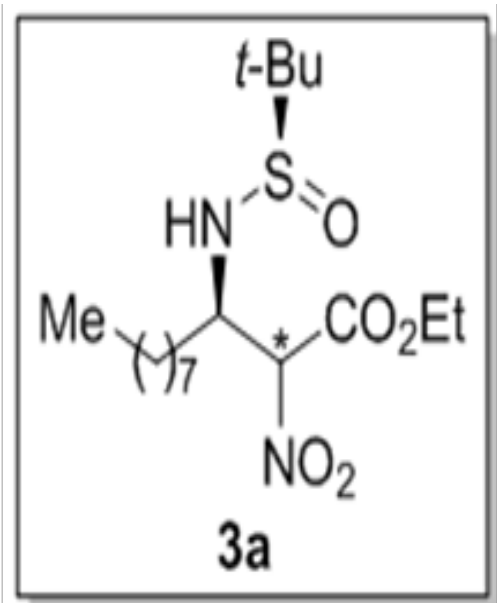

Figure 3 Ethyl $\left(2 \mathrm{R}^{*}, 3 \mathrm{R}, \mathrm{RS}\right)$-3-amino- $\mathrm{N}$-(tert-butanesulfinyl)-2nitroundecanoate (3a).

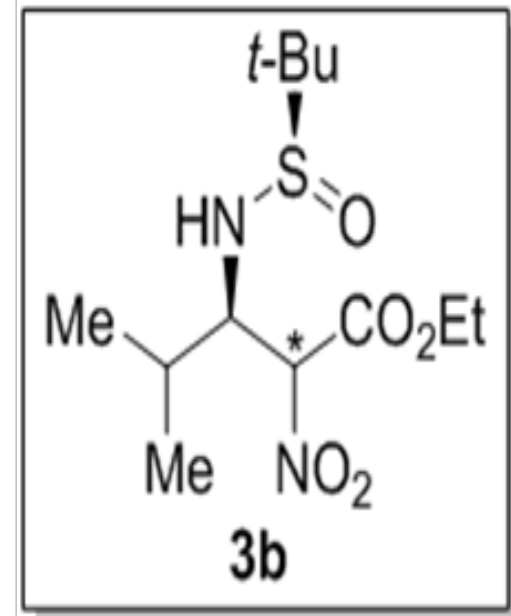

Figure 4 Ethyl (2R*,3R,RS)-3-amino-N-(tert-butanesulfinyl)-4-methyl-2nitropentanoate $(3 b)$.

Ethyl (2R*,3R, RS)-3-amino-N-(tert-butanesulfinyl)-5-methyl-2-nitrohexanoate (3c)

Yellow oil; $\mathrm{R}_{\mathrm{F}} 0.57$ (hexane/AcOEt 2:1); IR (film) v 2962, 2908, 2878, 1749, 1562, 1367, 1266, 1067, 1021, $734 \mathrm{~cm}^{-1}$; ${ }^{1} \mathrm{H}-\mathrm{NMR}$ $\left(300 \mathrm{MHz}, \mathrm{CDCl}_{3}\right) \delta 0.95-0.99\left(12 \mathrm{H}, \mathrm{m}, 4 \times \mathrm{CH}_{3}\right), 1.18\left[9 \mathrm{H}, \mathrm{s},\left(\mathrm{CH}_{3}\right)_{3}\right]$, $1.19\left[9 \mathrm{H}, \mathrm{s},\left(\mathrm{CH}_{3}\right)_{3}\right], 1.33\left(3 \mathrm{H}, \mathrm{t}, \mathrm{J}=7.1 \mathrm{~Hz}, \mathrm{CH}_{3}\right), 1.34(3 \mathrm{H}, \mathrm{t}, \mathrm{J}=7.1 \mathrm{~Hz}$ $\left.\mathrm{CH}_{3}\right), 1.66-1.89\left(6 \mathrm{H}, \mathrm{m}, 2 \times \mathrm{CH}, 2 \times \mathrm{CH}_{2}\right), 3.90(1 \mathrm{H}, \mathrm{d}, \mathrm{J}=9.6 \mathrm{~Hz}$, $\mathrm{NH}), 4.07(1 \mathrm{H}, \mathrm{d}, \mathrm{J}=9.6 \mathrm{~Hz}, \mathrm{NH}), 4.11-4.37\left(6 \mathrm{H}, \mathrm{m}, 2 \times \mathrm{CH}_{2}, 2 \times \mathrm{CH}\right)$, $5.21(1 \mathrm{H}, \mathrm{d}, \mathrm{J}=5.3 \mathrm{~Hz}, \mathrm{CH}), 5.28(1 \mathrm{H}, \mathrm{d}, \mathrm{J}=5.3 \mathrm{~Hz}, \mathrm{CH}) ;{ }^{13} \mathrm{C}-\mathrm{NMR}$ $\left(100 \mathrm{MHz}, \mathrm{CDCl}_{3}\right) \delta 13.8,13.9,21.5,21,9,22.4,22.45,22.5,22.6$, $24.1\left(\mathrm{CH}_{3}\right), 24.2,24.3(\mathrm{CH}), 41.5,42.6\left(\mathrm{CH}_{2}\right), 56.1(\mathrm{CH}), 56.5,56.6$ (C), 63.1, 63.2 (CH $), 89.5,90.9(\mathrm{CH}), 162.8,162.9(\mathrm{C})$; LRMS (EI) m/z159 (M+i-Bu-t-BuSO, 78), 142 (68), 113 (100), 96 (35), 85 (62), 57 (55) (Figure 5).

Ethyl (2R*, 3R, RS)-3-amino-N-(tert-butanesulfinyl)-2-nitro-5-phenylpentanoate (3d):

Yellow oil; $\mathrm{R}_{\mathrm{F}} 0.62$ (hexane/AcOEt 2:1); IR (film) v 3055, 3025,
2986, 2908, 2868, 1748, 1562, 1372, 1265, 1065, $733 \mathrm{~cm}^{-1}$; ${ }^{1} \mathrm{H}-\mathrm{NMR}$ $\left(300 \mathrm{MHz}, \mathrm{CDCl}_{3}\right) \delta 1.18\left[9 \mathrm{H}, \mathrm{s},\left(\mathrm{CH}_{3}\right)_{3}\right], 1.19\left[9 \mathrm{H}, \mathrm{s},\left(\mathrm{CH}_{3}\right)_{3}\right], 1.28$ $\left(3 \mathrm{H}, \mathrm{t}, \mathrm{J}=7.2 \mathrm{~Hz}, \mathrm{CH}_{3}\right), 1.29\left(3 \mathrm{H}, \mathrm{t}, \mathrm{J}=7.2 \mathrm{~Hz}, \mathrm{CH}_{3}\right), 1.95-2.00(2 \mathrm{H}, \mathrm{m}$, $2 \times \mathrm{CHH}), 2.14-2.25(2 \mathrm{H}, \mathrm{m}, 2 \times \mathrm{CHH}), 2.74-2.94\left(4 \mathrm{H}, \mathrm{m},, 2 \times \mathrm{CH}_{2}\right)$, 4.01-4.33 $\left(8 \mathrm{H}, \mathrm{m}, 2 \times \mathrm{CH}_{2}, 2 \times \mathrm{CH}, 2 \times \mathrm{NH}\right), 5.19(1 \mathrm{H}, \mathrm{d}, \mathrm{J}=5.3 \mathrm{~Hz}$, $\mathrm{CH}), 5.29(1 \mathrm{H}, \mathrm{d}, \mathrm{J}=5.3 \mathrm{~Hz}, \mathrm{CH}), 7.20-7.31(10 \mathrm{H}, \mathrm{m}, \mathrm{ArH}) ;{ }^{13} \mathrm{C}-\mathrm{NMR}$ $\left(100 \mathrm{MHz}, \mathrm{CDCl}_{3}\right) \delta 13.7,13.8,22.4,22.5\left(\mathrm{CH}_{3}\right), 31.6,31.7,34.1$, $35.0\left(\mathrm{CH}_{2}\right), 56.5,56.6(\mathrm{C}), 57.3,57.4(\mathrm{CH}), 63.1,63.2\left(\mathrm{CH}_{2}\right), 89.4$, $90.8(\mathrm{CH}), 126.3,128.3,128.4,128.6,128.65(\mathrm{CH}), 139.7,139.9(\mathrm{C})$, 162.6, 162.7 (C); LRMS (EI) m/z206 ( $\left.\mathrm{M}^{+}-\mathrm{C}_{7} \mathrm{H}_{7}-\mathrm{CO}_{2} \mathrm{Et}, 18\right), 141$ (13), 115 (18), 129 (22), 91 (100) (Figure 6).

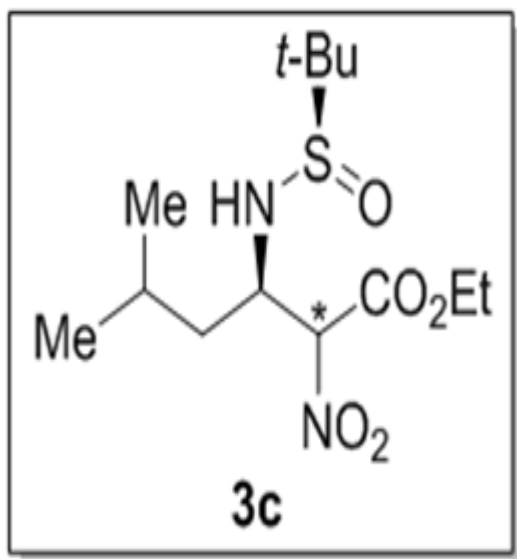

Figure 5 Ethyl $\left(2 R^{*}, 3 R, R S\right)-3$-amino- $N$-(tert-butanesulfinyl)-5-methyl-2nitrohexanoate (3c).

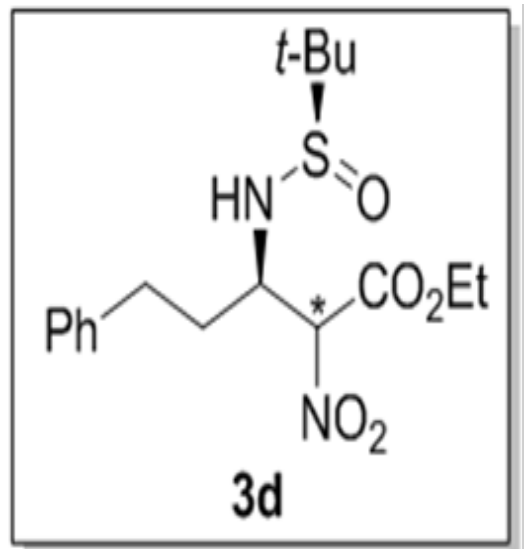

Figure 6 Ethyl (2R*3R,RS)-3-amino-N-(tert-butanesulfinyl)-2-nitro-5phenylpentanoate (3d).

Ethyl (2R*,3R, RS)-3-amino-N-(tert-butanesulfinyl)-2-nitro-4-phenylbutanoate (3e):

Yelow oil; $\mathrm{R}_{\mathrm{F}} 0.55$ (hexane/AcOEt 2:1); IR (film) v 3050, 2986, 1750, 1563, 1265, 1072, 1023, 732 $\mathrm{cm}^{-1}$; ${ }^{1} \mathrm{H}-\mathrm{NMR}\left(300 \mathrm{MHz}, \mathrm{CDCl}_{3}\right)$ $\delta 1.16\left[9 \mathrm{H}, \mathrm{s},\left(\mathrm{CH}_{3}\right)_{3}\right], 1.20\left[9 \mathrm{H}, \mathrm{s},\left(\mathrm{CH}_{3}\right)_{3}\right], 1.29(3 \mathrm{H}, \mathrm{t}, \mathrm{J}=7.1 \mathrm{~Hz}$, $\left.\mathrm{CH}_{3}\right), 1.34\left(3 \mathrm{H}, \mathrm{t}, \mathrm{J}=7.1 \mathrm{~Hz}, \mathrm{CH}_{3}\right), 2.90-3.01(2 \mathrm{H}, \mathrm{m}, 2 \times \mathrm{CHH}), 3.40-$ $3.50(2 \mathrm{H}, \mathrm{m}, 2 \times \mathrm{CHH}), 4.21-4.36\left(8 \mathrm{H}, \mathrm{m}, 2 \times \mathrm{CH}_{2}, 2 \times \mathrm{CH}, 2 \times \mathrm{NH}\right), 4.92$ $(1 \mathrm{H}, \mathrm{d}, \mathrm{J}=4.8 \mathrm{~Hz}, \mathrm{CH}), 5.03(1 \mathrm{H}, \mathrm{d}, \mathrm{J}=4.8 \mathrm{~Hz}, \mathrm{CH}), 7.16-7.38(10 \mathrm{H}$, m, ArH $) ;{ }^{13} \mathrm{C}-\mathrm{NMR}\left(100 \mathrm{MHz}, \mathrm{CDCl}_{3}\right) \delta 13.8,13.9,22.3,22.4\left(\mathrm{CH}_{3}\right)$, 40.1, $40.4\left(\mathrm{CH}_{2}\right), 56.7,56.8(\mathrm{C}), 59.4,59.6(\mathrm{CH}), 63.1,63.4\left(\mathrm{CH}_{2}\right)$, 87.4, 88.9 (CH), 127.5, 127.6, 129.1, 129.2, $129.4(\mathrm{CH}), 135.1(\mathrm{C})$, 162.7, 163.0 (C); LRMS (EI) m/z $191\left(\mathrm{M}+-\mathrm{C}_{7} \mathrm{H}_{7}{ }^{+}-\mathrm{C}_{4} \mathrm{H}_{8}, 5\right), 145$ (15), 117(100), 90 (29), 57 (18) (Figure 7). 


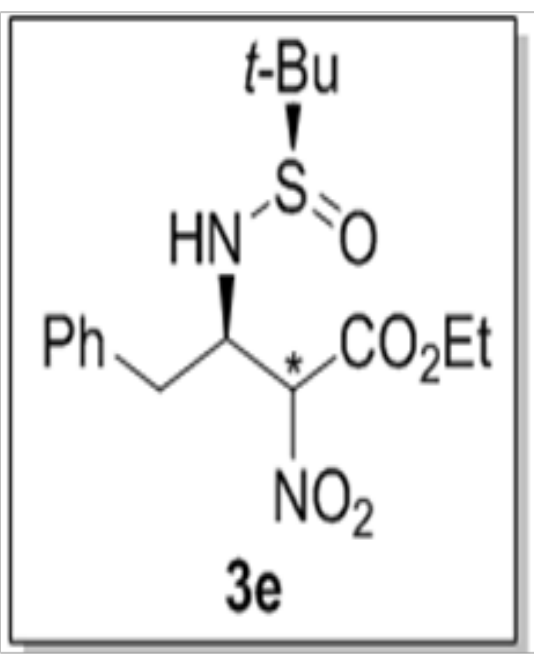

Figure 7 Ethyl (2R*,3R,RS)-3-amino-N-(tert-butanesulfinyl)-2-nitro-4phenylbutanoate (3e).

\section{Conclusion}

In conclusion, the addition of ethyl nitroacetate to chiral N-tertbutanesulfinyl aldimines can be performed under basic or acidic conditions, the process being very selective at the $\beta$-position but not at the $\alpha$-position, so the corresponding mixture of diastereomers is obtained.

\section{Acknowledgements}

We thank the continued financial support from our Ministerio de Ciencia e Innovación (MICINN; projects CTQ2010-20387, CONSOLIDER INGENIO 2010-CDS2007-00006, CTQ2011-24165), the Ministerio Economía y Competitividad (MINECO; projects CTQ2014-53695-P, CTQ2014-51912-RED), FEDER, Generalitat
Valenciana (PROMETEO 2009/039, PROMETEOII/2014/017) and the University of Alicante.

\section{Conflict of interest}

The author declares no conflict of interest.

\section{References}

1. Carreira EM, Kvaerno L. Classics in Stereoselective Synthesis. Germany: Wiley-VCH; 2009.

2. Westermann B. Asymmetric Catalytic Aza-Henry Reactions Leading to 1,2-Diamines and 1,2-Diaminocarboxylic Acids. Angew Chem Int Ed. 2003;42(2):151-153.

3. Davis FA, Zhou P, Chen BC. Asymmetric Synthesis of Amino Acids Using Sulfinimines (Thiooxime S-Oxides). Chem Soc Rev. 1998;27(1):13-18.

4. Robak MT, Herbage MA, Ellman JA. Synthesis and Applications of tertButanesulfinamide. Chem Rev. 2010;110(6):3600-3740.

5. García-Ruano JL, López-Cantanero J, De Haro T, et al. Preparation of $\alpha$-Amino Ketones, $\beta$-Amino Hydroxylamines Using Asymmetric AzaHenry Reactions of N-p-Tolylsulfinylimines with Nitroethane. Tetrahedron. 2006;62(52):12197-12203.

6. García-Muñoz MJ, Dema HK, Foubelo F, et al. Base-Promoted Diastereoselective Addition of Nitromethane and Nitroethane to N-tert-Butylsulfinyl Imines:Synthesis of N-Protected $\alpha$-Amino Acids and Amino Ketones. Tetrahedron: Asymmetry. 2014;25(4):362-372.

7. García-Muñoz MJ, Foubelo F, Yus M. Stereoselective Aza-Henry Reaction of Chiral N-tert-Butanesulfinyl Imines with Methyl and Ethyl 4 Nitrobutanoate: Easy Access to Enantioenriched 6-Substituted Piperidine-2,5-diones. Heterocycles. 2015;90(2):1419-1432.

8. Liu G, Cogan DA, Owens TD, et al. Synthesis of the Enantiomerically Pure N-tert-Butanesulfinyl Imines (tert-Butanesulfinimines) by Direct Condensation of tert-Butanesulfinamide with Aldehydes and Ketones. $J$ Org Chem. 1999;64(4):1278-1284. 